\title{
Free oscillation rheometry monitoring of haemodilution and hypothermia and correction with fibrinogen and factor XIII concentrates
}

\author{
Dag Winstedt ${ }^{*}$, Nahreen Tynngård ${ }^{2,3}$, Knut Olanders $^{1}$ and Ulf Schött ${ }^{1}$
}

\begin{abstract}
Background: Haemodilution and hypothermia induce coagulopathy separately, but their combined effect on coagulation has not been widely studied. Fibrinogen concentrate can correct dilutional coagulopathy and has an additional effect when combined with factor XIII concentrate. However, their effect on dilutional coagulopathy concomitant with hypothermia has not been studied previously. Free oscillation rheometry - FOR (Reorox ${ }^{\circledR}$ ) - is a novel viscoelastic haemostatic assay that has not been studied in this context before.

Methods: Blood from 10 healthy volunteers was diluted by 33\% with hydroxyethyl starch or Ringer's acetate solutions. Effects of fibrinogen added in vitro with and without factor XIII were studied at $33^{\circ} \mathrm{C}$ and $37^{\circ} \mathrm{C}$. Coagulation velocity (coagulation time) and clot strength (elasticity) were assessed with FOR. Coagulation was initiated in vitro with thromboplastin alone, or thromboplastin plus a platelet inhibitor.

Results: Hydroxyethyl starch increased the coagulation time and decreased clot strength significantly more than Ringer's acetate solution, both in the presence and absence of a platelet inhibitor. There was a significant interaction between haemodilution with hydroxyethyl starch and hypothermia, resulting in increased coagulation time. After addition of fibrinogen, coagulation time shortened and elasticity increased, with the exception of fibrinogen-dependent clot strength (i.e., elasticity in the presence of a platelet inhibitor) after hydroxyethyl starch haemodilution. Factor XIII had an additional effect with fibrinogen on fibrinogen-dependent clot strength in blood diluted with Ringer's acetate solution. Hypothermia did not influence any of the coagulation factor effects.

Conclusions: Both haemodilution and mild hypothermia impaired coagulation. Coagulopathy was more pronounced after haemodilution with hydroxyethyl starch than with Ringer's acetate. Addition of fibrinogen with factor XIII was unable to reverse hydroxyethyl starch induced clot instability, but improved coagulation in blood diluted with Ringer's acetate solution. Fibrinogen improved coagulation irrespective of hypothermia.
\end{abstract}

Keywords: Free oscillation rheometry, Thrombelastography, Coagulation factor concentrate, Fibrinogen, Factor XIII, Haemodilution, Hypothermia, Coagulopathy, Hydroxyethyl starch, Ringer's acetate solution

\section{Introduction}

Exsanguination is the second most common cause of death in major trauma after central nervous system (CNS) injury $[1,2]$. To maintain an adequate circulatory blood volume and oxygen carrying capacity, traumatic and surgical haemorrhages are generally initially compensated for by administrating a combination of

\footnotetext{
* Correspondence: dag.winstedt@med.lu.se

${ }^{1}$ Consultant Anaesthetist, Lund University, Skane Universisty Hospital, Lund, 22185 Lund, Sweden

Full list of author information is available at the end of the article
}

crystalloids, colloid solutions and packed red blood cells (PRBC). However, this results in haemodilution, hypothermia and acidosis, thus promoting the coagulopathy often seen in major haemorrhage [3,4]. In addition, synthetic colloid solutions such as hydroxyethyl starch affect coagulation more than crystalloid solutions do $[5,6]$.

Fibrinogen is the first coagulation factor to reach critical levels in major haemorrhage [7] and fibrinogen concentrate should be given in active bleeding when plasma levels reach 1.5 to $2.0 \mathrm{~g} / \mathrm{l}$ [8]. This increases clot stability

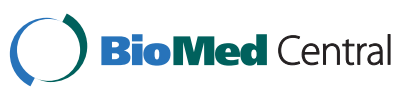


after haemodilution $[9,10]$, reduces bleeding [11-13] and may improve survival $[14,15]$. The benefit of fibrinogen concentrate may be augmented by factor XIII (FXIII), since FXIII is responsible for cross-linking fibrin monomers [16].

Viscoelastic haemostatic assays (VHA), such as thrombelastography (TEG) and rotational thrombelastometry (ROTEM), may better guide blood component therapy in major bleeding compared to traditional coagulation tests $[4,17]$. According to analysis with VHAs, coagulation is impaired by both hypothermia $[18,19]$ and haemodilution. In this study, we analysed coagulation with free oscillation rheometry (FOR), a novel viscoelastic haemostatic assay. The aim of our study was to investigate hypothermiaand haemodilution-induced coagulopathy measured with free oscillation rheometry, and to what extent this coagulopathy could be reversed with fibrinogen concentrate, combined with or without FXIII. Our hypotheses were that hypothermia and haemodilution would each impair coagulation measured with FOR and that the combination of hypothermia and haemodilution would interact, that is would impair coagulation synergistically. We also hypothesised that fibrinogen could reduce this coagulopathy, with an additional effect of FXIII.

\section{Materials and methods}

\section{Study subjects and sampling}

Ten healthy individuals without any known coagulation disorder (one woman and nine men, average age 43 years, with a mean haemoglobin level of $150 \mathrm{~g} / \mathrm{l}$ ) gave their written informed consent to donate $50 \mathrm{ml}$ of blood. None of the subjects had taken any medication, including naturopathic preparations, during the seven days before blood collection. The Ethical Board, Lund, Sweden, approved the study (Registration Number: DNR 484).

Venipuncture was performed with a $21 \mathrm{G}$ needle and blood was collected into plastic Vacutainers ${ }^{\circledR}$, mixing one part of citrate with nine parts of blood (BD, Plymouth, UK (citrate $0.129 \mathrm{mmol} / \mathrm{l})$ ). Blood was collected on two occasions on the same day per individual. The tubes were incubated for a minimum of 30 minutes and a maximum of four hours at a temperature of $33^{\circ}$ or $37^{\circ} \mathrm{C}$ to ensure temperature equilibration.

\section{Haemodilution}

Blood was diluted 33\% with either Ringer's acetate solution (RA) (Fresenius Kabi, Bad Homburg, Germany) or with 6\% hydroxyethyl starch in saline (HES) (Mw $130 \mathrm{kDa}$, substitution 0.42, B. Braun, Melsungen, Germany). The solutions had a temperature of $33^{\circ}$ or $37^{\circ} \mathrm{C}$ and the haemodiluted samples were kept at a temperature of $33^{\circ}$ or $37^{\circ} \mathrm{C}$ until coagulation was analysed.

\section{Coagulation factor concentrates}

Fibrinogen concentrate (Haemocomplettan ${ }^{\circledR}$, now renamed Riastap $^{\circledR}$, CSL Behring, Marburg, Germany) was dissolved in distilled water (Fresenius Kabi) to a concentration of 20 g/l. FXIII concentrate (Fibrogammin ${ }^{\circledR}$, CSL Behring) was dissolved in the solvent supplied by the manufacturer to a concentration of $62.5 \mathrm{U} / \mathrm{ml} .120 \mu \mathrm{l}$ of fibrinogen alone or $120 \mu \mathrm{l}$ of fibrinogen supplemented with $15 \mu \mathrm{l}$ of FXIII were added to a total sample volume of $3000 \mu$ l. These amounts correspond to therapeutic doses used in clinical practice: $4 \mathrm{~g}$ fibrinogen and $1550 \mathrm{U}$ of FXIII to a $70-\mathrm{kg}$ man, i.e., $55 \mathrm{mg}$ fibrinogen and $22 \mathrm{U}$ of FXIII per $\mathrm{kg}$ of body weight [20].

\section{Coagulation analysis}

Clot formation and clot strength was studied with free oscillation rheometry (FOR), assessed with the ReoRox G2 ${ }^{\circledR}$ rheometer (Medirox AB, Nyköping, Sweden). FOR, like thrombelastography, utilizes an oscillating movement to monitor coagulation. The sample is added to a reaction chamber, which consists of a gold-coated sample cup with a gold-coated cylinder (bob) suspended in the blood sample [21]. FOR uses a torsion wire system to set the sample into oscillation (Figure 1). A magnet pulls back the measuring head connected to the torsion wire. On release, the torsion wire will set the cup into free oscillation and its movement is recorded by an optical detector. The changes of damping and frequency of the oscillation correlates to viscosity and elasticity respectively, which are recorded as a viscosity curve and an elasticity curve (Figure 1 ).

The coagulation time (COT) is obtained from the viscosity curve. Coagulation time 1 (COT1) is the time to the start of clot formation when the initial strands of fibrin are formed [22]. Coagulation time 2 (COT2) is the time to complete clot formation after which elasticity starts building up and is equivalent to CT time for ROTEM. The clot strength in terms of maximum elasticity (G'max) is determined from the elasticity curve [23] and G'max is equivalent to MCF for ROTEM.

All analyses were performed according to the manufacturer's instructions. Sample cups were placed into the heating blocks of the apparatus which were set to either $33^{\circ}$ or $37^{\circ} \mathrm{C}$. Temperatures were allowed to equilibrate and $1000 \mu \mathrm{l}$ of blood sample was added to each sample cup. After re-calcification with $25 \mu \mathrm{l}$ of $0.5 \mathrm{M} \mathrm{CaCl}_{2}$ (Medirox $\mathrm{AB})$, coagulation was initiated with thromboplastin alone (FibScreen1, Medirox AB) or thromboplastin in the presence of abciximab (FibScreen2). Abciximab is a glycoprotein IIb/IIIa-receptor antibody and thus, inhibits platelet interaction with fibrinogen. Accordingly, FibScreen2 (Fib2) provided information about the functional fibrinogen concentration and fibrin stability of the clot. FOR tracings were analyzed for COT1, COT2 and G'max. G'max was 

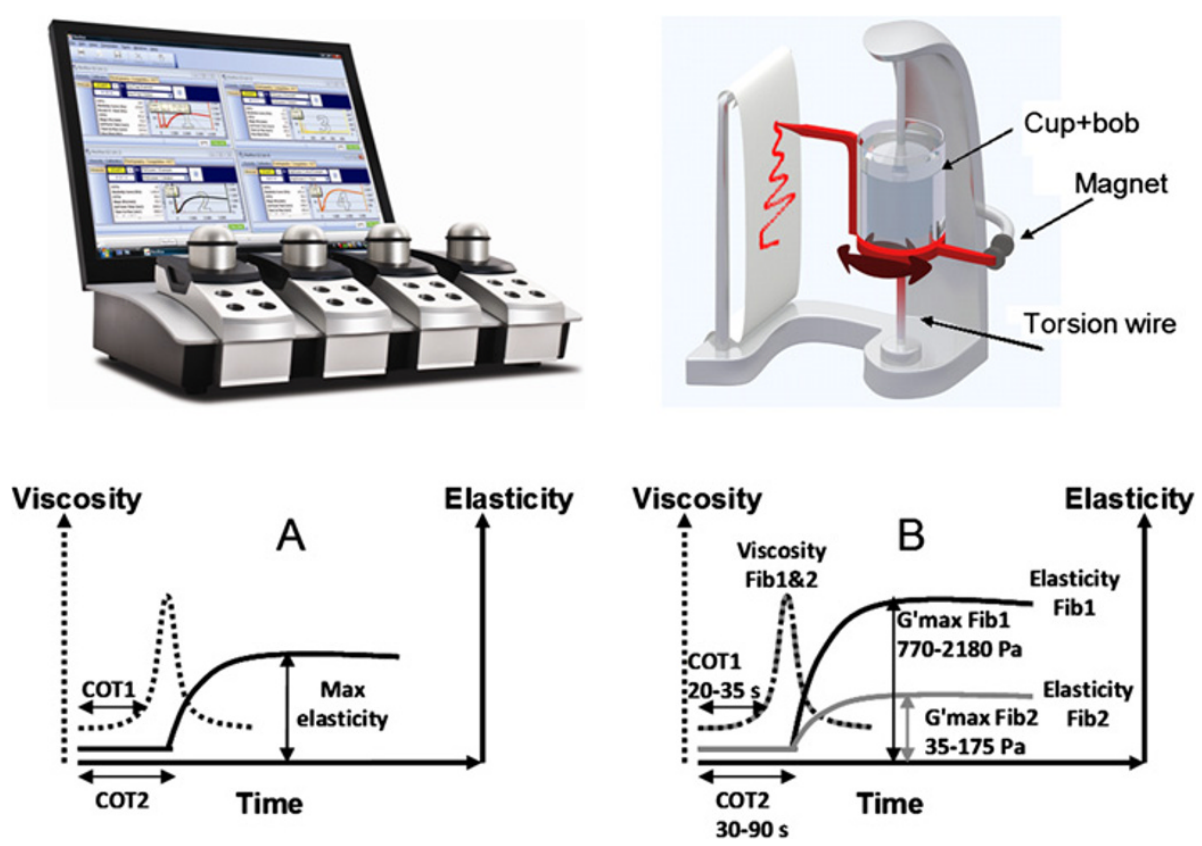

Figure 1 Free oscillation rheometry. The ReoRox G2 rheometer (upper left corner) and a schematic picture of its free oscillation sample cup (upper right corner). The magnet turns the sample cup around the torsion wire. Upon release, an oscillatory movement starts which is recorded by an optical detector. The change in damping generates a viscosity curve (dashed line) and the change in frequency generates an elasticity curve (full line) as shown in $\mathbf{A}$. The damping (viscosity) of the oscillation increases as the sample coagulates until all is coagulated at which point the viscosity returns to baseline since the damping of the oscillation will not be affected anymore. This is followed by an increase in oscillation frequency (elasticity) when the platelets retract the clot. The height of the elasticity curve represents the strength of the clot. Variables detected are indicated with arrows (COT1- time to beginning of clot formation, COT2 - time to complete clot formation and Max elasticity (G'max) maximum clot strength). B shows the differences in elasticity between FibScreen1 (full black line) and FibScreen2 (full grey line). The viscosity (COT1 and COT2) for FibScreen1 (dashed grey line) and FibScreen2 (dashed dotted line) is similar in a normal sample. Normal ranges are presented in the figure.

determined for whole blood activated with FibScreen1 (Fib1) and for whole blood with inhibited platelets using Fib2 as activator. The difference in G'max between Fib1 and Fib2 was calculated as a measure of platelet-dependent clot strength. The normal ranges for the FOR parameters according to the manufacturer are presented in Figure 1. The coefficients of variation (CV) for the FOR parameters were 3\% for COT1, 9\% for COT2, 11\% for Fib1 G'max and 15\% for Fib2 G'max. The lowest detection level of G'max is set to $10 \mathrm{~Pa}$ in the software, which is well above the normal range for Fib2 G'max, and thus FOR should be able to detect low levels of functional fibrinogen concentrations.

\section{Statistical analysis}

Kolmogorov-Smirnov tests were significant for several variables, showing that the data were not normally distributed. For this reason, all data were transformed, using the aligned rank transformation [24]. All transformed data were further analysed with repeated measures analysis of variance (ANOVA). Two separate ANOVAs were performed: a twoway ANOVA to study the influence of temperature and different solutions on coagulation and a three-way ANOVA to study the influence of added coagulation factors in hypothermia and haemodilution. Effects were presented as estimated marginal means, i.e., the mean of a factor averaged across all levels of the other factors. Where statistically significant differences were detected, further investigations of differences were made with contrast analysis. The Bonferroni correction was used to adjust for multiple comparisons. The aligned rank transformation was calculated with the free software ARTool. ANOVA statistical analysis was performed using PASW 18 (SPSS). Data were considered significant when the P-value was $<0.05$.

\section{Results}

FOR in hypothermia and haemodilution

In the first part of this study, the effects of mild hypothermia and haemodilution, alone or in combination, were investigated.

\section{Hypothermia}

Mild hypothermia impaired all the measured aspects of coagulation (Table 1 and Figure 2). Both the time to the initiation phase (COT1) of coagulation and the time to complete clot formation (COT2) were significantly 
Table 1 Effects of hypothermia and haemodilution on coagulation measured with free oscillation rheometry (FOR)

\begin{tabular}{|c|c|c|c|c|c|c|c|c|c|}
\hline \multirow{3}{*}{$\frac{\text { Variable }}{\text { COT1 (s) }}$} & \multicolumn{3}{|c|}{ Temperature } & \multicolumn{6}{|c|}{ Solution } \\
\hline & \multirow{2}{*}{$\begin{array}{r}37^{\circ} \mathrm{C} \\
18.1\end{array}$} & \multicolumn{2}{|c|}{$33^{\circ} \mathrm{C}$} & \multirow{2}{*}{$\begin{array}{r}\text { Undiluted } \\
17.6\end{array}$} & \multicolumn{2}{|c|}{ RA } & \multicolumn{3}{|c|}{ HES } \\
\hline & & 21.2 & $* *$ & & 20.1 & $* *$ & 21.3 & **** & \\
\hline COT2 (s) & 58.3 & 68.8 & $* * *$ & 48.5 & 55.5 & $* *$ & 86.7 & $* *$ & $t$ \\
\hline G'max Fib1 (Pa) & 716 & 613 & $* * *$ & 983 & 617 & $* * *$ & 395 & $* * *$ & t十+ \\
\hline G'max Fib2 (Pa) & 31.7 & 24.5 & $* * *$ & 43.3 & 29.5 & $* * *$ & 11.5 & $* * *$ & t十+ \\
\hline G'max Fib1-2 (Pa) & 685 & 589 & $* *$ & 940 & 587 & $* * *$ & 383 & $* * *$ & t十+ \\
\hline
\end{tabular}

Effects on coagulation of hypothermia $\left(33^{\circ} \mathrm{C}\right.$ versus $\left.37^{\circ} \mathrm{C}\right)$ and $33 \%$ haemodilution with RA or HES compared to undiluted blood, presented as estimated marginal means. COT1 and COT2 are coagulation time 1 and 2, respectively. The maximal clot strength is presented as G'max for Fib1 (without platelet inhibition) and G'max for Fib2 (with platelet inhibition). Platelet-dependent clot strength is presented by the difference between G'max for Fib1 and that for Fib2, denoted as G'max Fib1-2.

** and ${ }^{* * *}$ stands for $p<0.01$ and $p<0.001$, respectively, when the two temperatures were compared or when diluted blood (RA or HES) was compared to undiluted blood. $+\dagger$ and $+\dagger \dagger$ stands for $p<0.01$ and $p<0.001$, respectively, when haemodilution with RA was compared to HES. See text for details on interactions. $\mathrm{N}=10$.
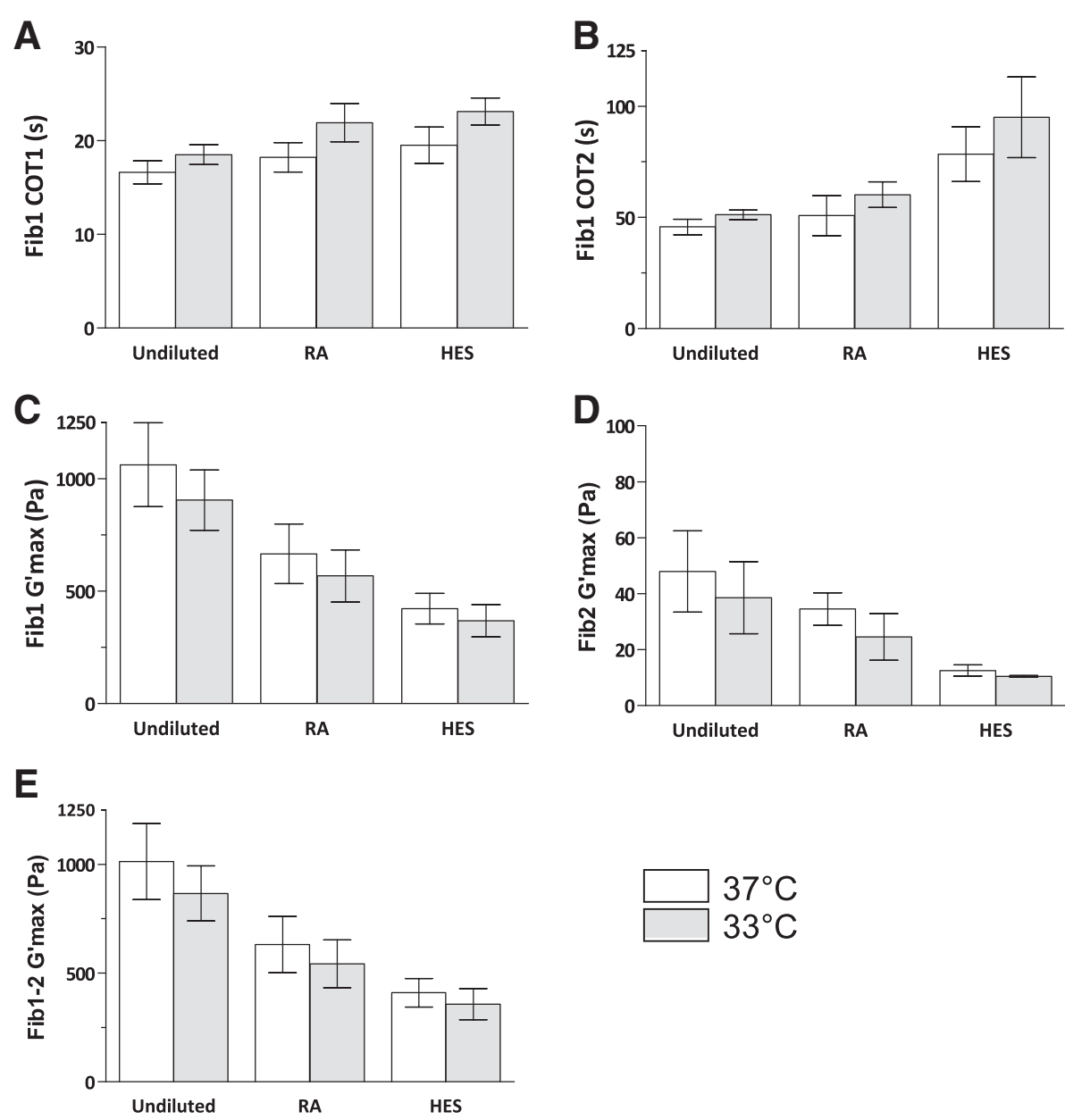

Figure 2 Effects of haemodilution and hypothermia on coagulation. Coagulation of undiluted, RA-diluted and HES-diluted blood at 33 and $37^{\circ} \mathrm{C}$ as assessed by FOR. The parameters COT1 (A), COT2 (B), maximum elasticity for FibScreen1 (C), maximum elasticity for FibScreen2 (D) and the difference in maximum elasticity for FibScreen 1 and 2 (E) are shown. Coagulation time 1 (COT1) represents the beginning of clot formation and coagulation time 2 (COT2) a fully formed clot. G'max is the maximum elasticity of the fully formed clot. FibScreen1 (Fib1) denotes that thromboplastin was added to the sample and FibScreen2 (Fib2) indicates that thromboplastin and abciximab were added, the latter a potent platelet inhibitor. Thus, Fib1 G'max represents whole blood clot strength and Fib2 G'max fibrinogen-dependent clot strength. FS1 - FS2 G'max was calculated to represent platelet-dependent clot strength. $\mathrm{N}=10$. Bars are $95 \% \mathrm{Cl}$. 
prolonged in hypothermia. Also, the final clot strength weakened with hypothermia, measured as a decrease in G'max. G'max decreased in whole blood (Fib1), in whole blood with inhibited platelets (Fib2), as well as the calculated platelet-dependent (the difference between Fib1 and Fib2) G'max.

\section{Haemodilution}

Haemodilution with RA or HES impaired all the measured aspects of coagulation (Table 1 and Figure 2). Coagulation times (COT1 and COT2) were prolonged and G'max decreased in whole blood (Fib1), in whole blood with inhibited platelets (Fib2), as well as the calculated plateletdependent clot strength. Haemodilution with HES consistently impaired coagulation more than haemodilution with RA. However, this difference was not statistically significant for COT1 (Table 1).

\section{Interaction of hypothermia and haemodilution}

A significant interaction effect (synergy) was shown for COT2 between hypothermia and haemodilution with HES compared to undiluted blood $(\mathrm{p}=0.035$; hypothermia $\mathrm{X}$ haemodilution (undiluted vs. HES)). COT2 was prolonged after haemodilution with HES and even more when HES haemodilution was combined with hypothermia (Figure 2). Thus, hypothermia and haemodilution with HES prolonged COT2 more than explained by their respective additive effects. Interestingly, this synergy could not be found between hypothermia and haemodilution with RA.

Significant interaction effects were also shown for Fib2 G'max between hypothermia and haemodilution with HES compared to either undiluted blood $(\mathrm{p}<0.001)$ or blood diluted with RA ( $\mathrm{p}=0.003$; Table 2$)$. However, these statistically significant interactions were not proofs of synergy. Instead they showed, as seen in Figure 2, that hypothermia had less additional effect on Fib2 G'max in blood diluted with HES, as compared to undiluted blood or blood diluted with RA. The reason for this is probably technical, since the FOR instrument never yields G'max values of $<10 \mathrm{~Pa}$. After haemodilution with HES, FOR is simply unable to detect any additional decreases in Fib2 G'max.

\section{FOR in hypothermia and haemodilution with coagulation factor substitution}

In the second part of this study, the effects on coagulation of adding factor concentrate (fibrinogen with or without FXIII) in hypothermia and haemodilution were studied.

\section{Coagulation factor substitution}

The overall effect of substitution with coagulation factors is presented in Table 3 and Figure 3. The addition of fibrinogen significantly improved all FOR parameters, except the initiation of coagulation (COT1). However, a small additional effect when combining fibrinogen with FXIII shortened COT1 significantly compared to the control group without coagulation factors added. Apart from this effect of FXIII on COT1, fibrinogen-dependent clot strength, i.e., Fib2 G'max was the only parameter to benefit further by combining fibrinogen with FXIII. Thus, Fib2 G'max increased significantly after adding fibrinogen and rose significantly more when fibrinogen was combined with FXIII. However, as seen in Figure 3, Fib2 G'max increased with the addition of fibrinogen (with or without FXIII) only in RA-diluted blood (see below).

\section{Interaction of type of solution in haemodilution and addition of coagulation factor}

There were significant interactions between the type of solution in haemodilution and added coagulation factor for COT2 and Fib2 G'max.

Adding fibrinogen $(\mathrm{p}=0.001)$ or fibrinogen with FXIII $(\mathrm{p}=0.002)$ decreased COT2 more in blood diluted with

Table 2 Interaction effects of (a) temperature and the type of solution in haemodilution and (b) the type of solution in haemodilution and added coagulation factor

\begin{tabular}{|c|c|c|c|c|c|c|}
\hline & \multicolumn{3}{|c|}{ (a) Temperature $X$ solution } & \multicolumn{3}{|c|}{ (b) Solution $X$ coagulation factor } \\
\hline & Und & Und. & RA & Control vs. & Control vs. & Fibrinogen vs. \\
\hline Variable & vs. RA & vs. HES & vs. HES & Fibr. & Fibr. + FXIII & Fibr. + FXIII \\
\hline COT1 (s) & - & - & - & - & - & - \\
\hline $\operatorname{coT} 2(s)$ & - & 0.035 & - & 0.001 & 0.002 & - \\
\hline G'max Fib1 (Pa) & - & - & - & - & - & - \\
\hline G'max Fib2 (Pa) & - & $<0.001$ & 0.003 & $<0.001$ & $<0.001$ & $<0.001$ \\
\hline G'max Fib1-2 (Pa) & - & - & - & - & - & - \\
\hline \multicolumn{7}{|c|}{$\begin{array}{l}\text { Significances ( } \mathrm{p} \text {-values) of the interaction effects. The left half of the table (a) shows the interaction between temperature }\left(33^{\circ} \mathrm{C} \text { versus } 37^{\circ} \mathrm{C} \text { ) and solution }\right. \\
\text { (Und. = undiluted, RA, or HES), according to a two-way ANOVA (Temperature X Solution). } \\
\text { The right half of the table (b) shows the interaction between solution (RA or HES) and added coagulation factor (Control, i.e., no addition of coagulation factor, } \\
\text { fibrinogen or fibrinogen with FXIII), according to a three-way ANOVA (Temperature X Solution X Coagulation Factor). Not shown from the latter ANOVA are the } \\
\text { interactions of Temperature X Coagulation Factor. None of these interactions were significant. The maximal clot strength is presented as G'max for Fib1 (without } \\
\text { platelet inhibition) and G'max for Fib2 (with platelet inhibition). Platelet-dependent clot strength is presented by the difference between G'max for Fib1 and that } \\
\text { for Fib2, denoted as G'max Fib1-2. RA is Ringer's acetate solution. HES is hydroxyethyl starch in saline. Interactions marked with - were not significant. N = 10. }\end{array}$} \\
\hline
\end{tabular}


Table 3 Effects of added coagulation factors on coagulation measured with free oscillation rheometry (FOR)

\begin{tabular}{|c|c|c|c|c|c|c|}
\hline \multirow{3}{*}{$\frac{\text { Variable }}{\text { COT1 (s) }}$} & \multicolumn{6}{|c|}{ Coagulation Factor } \\
\hline & \multirow{2}{*}{$\begin{array}{r}\text { Control } \\
20.7\end{array}$} & \multicolumn{2}{|c|}{ Fibrinogen } & \multicolumn{3}{|c|}{ Fibrinogen + FXIII } \\
\hline & & 19.4 & & 19.0 & $* *$ & \\
\hline cOT2 (s) & 71.1 & 55.2 & $* * *$ & 53.1 & $* * *$ & \\
\hline G'max Fib1 (Pa) & 506 & 585 & $* *$ & 606 & $* *$ & \\
\hline G'max Fib2 (Pa) & 20.5 & 31.0 & $* * *$ & 36.3 & $* * *$ & †† \\
\hline G'max Fib1-2 (Pa) & 485 & 554 & $* *$ & 570 & $* *$ & \\
\hline
\end{tabular}

Effects on coagulation of the coagulation factors fibrinogen, fibrinogen with factor XIII (Fibrinogen + FXIII) or no addition of coagulation factor (Control). Data are estimated marginal means. COT1 and COT2 are coagulation time 1 and 2, respectively. The maximal clot strength is presented as G'max for Fib1 (without platelet inhibition) and G'max for Fib2 (with platelet inhibition). Platelet-dependent clot strength is presented by the difference between G'max for Fib1 and that of Fib2, denoted as G'max Fib1-2. ** and *** stands for $p<0.01$ and $p<0.001$, respectively, when the addition of coagulation factor was compared to the control group. †† and ††† stands for $\mathrm{p}<0.01$ and $p<0.001$, respectively, when the addition of fibrinogen alone was compared to the addition of fibrinogen with FXIII. See text for details on interactions. $\mathrm{N}=10$.

HES than with RA. There was no significant difference between fibrinogen and fibrinogen with FXIII.

On the contrary, Fib2 G'max increased more after adding fibrinogen $(\mathrm{p}<0.001)$ or fibrinogen with FXIII $(\mathrm{p}<0.001)$ in blood diluted with RA than in that with HES. In fact, Fib2 G'max did not improve at all in blood diluted with HES (Figure 3). In RA diluted blood, Fib2 G'max increased most when fibrinogen was combined with FXIII $(\mathrm{p}<0.001)$.

\section{Interaction of hypothermia and addition of coagulation factor}

There were no significant interactions between hypothermia and the addition of any combination of coagulation factors.

\section{Discussion}

This study shows that cooling blood to $33^{\circ} \mathrm{C}$ and haemodilution with HES interacts to impair clot formation. We also found that fibrinogen substitution was effective also at $33^{\circ} \mathrm{C}$, alone or in combination with haemodilution. The type of solution in haemodilution, however, did affect the reversal effect of fibrinogen in dilutional coagulopathy. After adding fibrinogen, clot propagation (COT2) improved more in HES haemodilution, while fibrinogendependent clot strength (Fib2 G'max) increased in RA haemodilution only, and not at all in HES haemodilution. Furthermore, combining fibrinogen with FXIII had an additional effect on fibrinogen-dependent clot strength (Fib2 G'max) with RA haemodilution only. Apart from these principle findings, we also confirmed the results of previous studies with other viscoelastic haemostatic assays that showed that both hypothermia and haemodilution independently impaired coagulation, and that HES impaired coagulation more than RA.

VHAs such as thrombelastography, measure coagulation in whole blood rather than in plasmaand they are therefore able to detect interactions between platelets, red blood cells and coagulation factors. Thus, VHAs more accurately describe coagulation in vivo and they are better predictors of bleeding than traditional measures of coagulation [25]. FOR's ability to measure viscosity changes enables detection of the start of clot formation (COT1), which is in contrast to with thrombelastography. FOR uses free oscillation, which results in less strain on the clot as compared with thrombelastography. Compared to maximal clot strength measured with thrombelastography, the corresponding measure with FOR (Fib1 G'max) is more dependent on platelets than on factors affecting the fibrinogen polymerization, i.e. fibrinogen concentration and FXIII [21]. Thus, FOR may provide new information on the effect of hemodilution and hypothermia on coagulation.

In concordance with earlier studies with VHAs, we found that hypothermia impaired clot formation variables $[18,26]$. In addition, we observed that clot strength was decreased even by mild hypothermia, which other studies failed to detect $[19,27]$. Previous studies only noted this decrease in clot strength during moderate to severe hypothermia $[18,26]$. The different measuring principles of ROTEM and FOR might be the cause of the different effects observed on clot strength by hypothermia. The possible higher sensitivity of clot strength variables measured with the FOR than with other methods is interesting. However, the decrease in clot strength observed from $37^{\circ}$ to $33^{\circ} \mathrm{C}$ is moderate and unlikely to have any clinical implications.

Our results demonstrated that haemodilution with either HES or RA compromised coagulation, and that the effect of HES was more pronounced. This is in accordance with previous studies where HES was shown to affect coagulation more than RA both in vitro $[28,29]$ and in vivo [30,31]. It is believed that HES, in contrast to crystalloids, also impair fibrinogen/fibrin polymerization [29].

Not only platelets are important cellular components of global haemostasis, but also erythrocytes play an important role [32]. The role of the haematocrit level has been previously studied. In vitro studies with FOR and TEG, where the platelet counts were held constant, showed increased clot strengths with decreasing haematocrit levels $[21,33]$. This increase in clot strength may be due to increasing fibrinogen levels after haemodilution with plasma. Ogawa recently also showed an increase in clot strength after haemodilution with plasma [34]. However, at a given fibrinogen concentration, clot strength instead improved with falling haematocrit levels. Thus, fibrinogen 

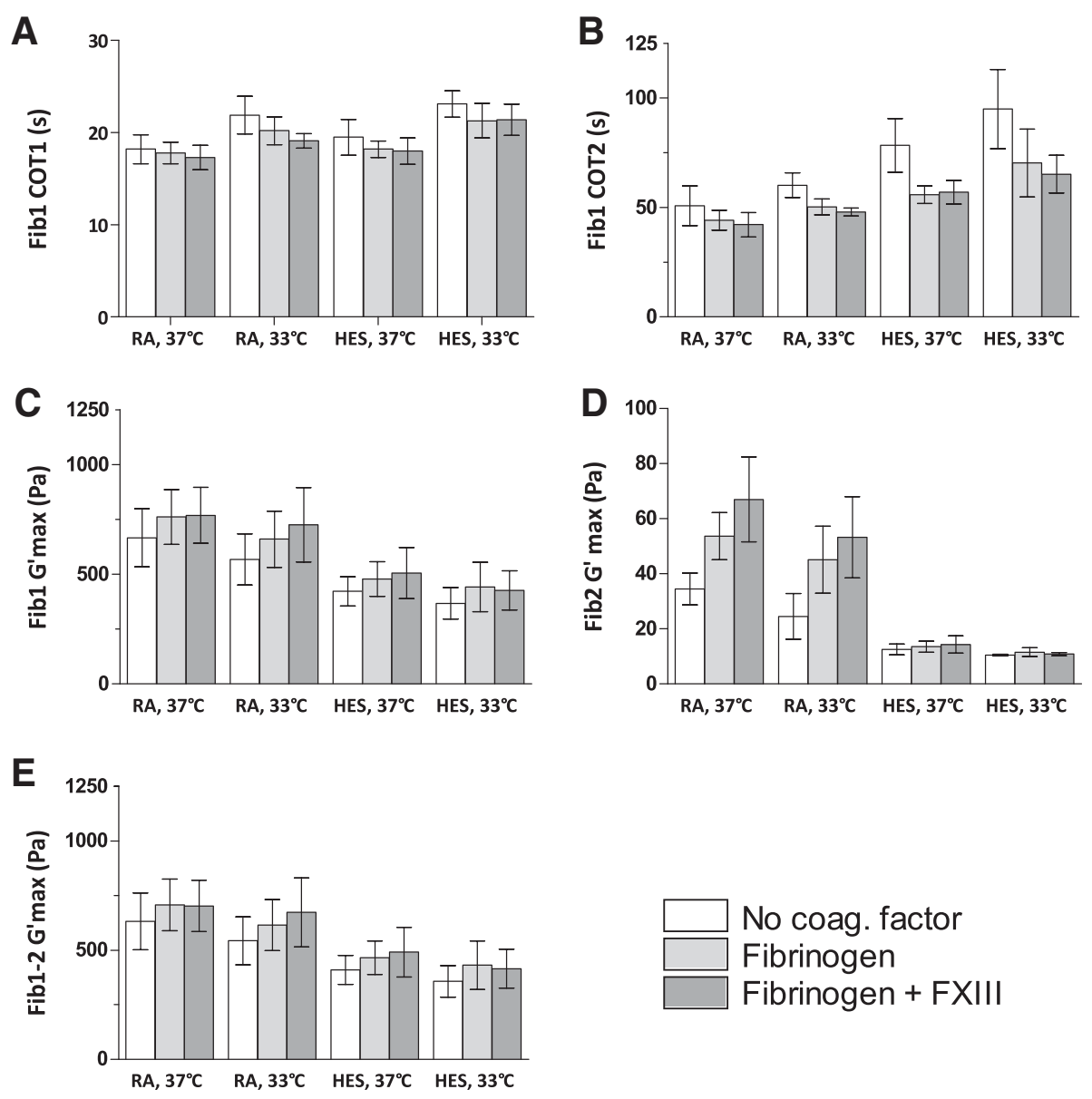

No coag. factor

Fibrinogen

Fibrinogen + FXIII

Figure 3 Effects of factor concentrate addition on coagulation. Coagulation of RA-diluted and HES-diluted blood at 33 and $37^{\circ} \mathrm{C}$ upon addition of factor concentrates (fibrinogen or fibrinogen + FXIII) as assessed by FOR. The parameters COT1 (A), COT2 (B), maximum elasticity for FibScreen1 (C), maximum elasticity for FibScreen2 (D) and the difference in maximum elasticity for FibScreen 1 and 2 (E) are shown. Coagulation time 1 (COT1) represents the beginning of clot formation and coagulation time 2 (COT2) a fully formed clot. G'max is the maximum elasticity of the fully formed clot. FibScreen1 (Fib1) denotes that thromboplastin was added to the sample and FibScreen2 (Fib2) indicates that

thromboplastin and abciximab were added, the latter a potent platelet inhibitor. Thus, Fib1 G'max represents whole blood clot strength and Fib2 G'max fibrinogen-dependent clot strength. FS1 - FS2 G'max was calculated to represent platelet-dependent clot strength. $\mathrm{N}=10 . \mathrm{Bars}$ are $95 \% \mathrm{Cl}$.

levels seem to be far more important than haematocrit level for the final clot strength.

Other studies have not found any interaction effects between hypothermia and haemodilution, as assessed by coagulation time and clot strength [35,36]. We discerned no interactions for the clot strength variables, but we did find significant interaction effects between hypothermia and haemodilution with HES in the time to complete clot formation (COT2). There was also a tendency towards interaction between hypothermia and haemodilution with RA for the time to complete clot formation (COT2), as well as between hypothermia and haemodilution with both solutions for the initiation of clot formation (COT1). FOR and thrombelastography are not completely comparable methods. For example, in contrast to thrombelastography, FOR can measure viscosity changes and discerns the coagulation time from the changes in viscosity. This may explain the inability of thrombelastography to detect any interaction effects on variables describing the early phases of coagulation.

Substitution with fibrinogen concentrate in dilutional coagulopathy has been demonstrated to effectively increase clot strength $[9,10,13]$ and to reduce bleeding $[11,37]$. A high ratio of fibrinogen to red blood cell transfusion is associated with improved survival rates [38]. In this study, we found that fibrinogen increased clot strength and shortened the coagulation time (COT1 and COT2). The reduction in COT1, though, was very small and significant only when fibrinogen was combined with FXIII. These results are in agreement with the aforementioned studies with ROTEM, where addition of fibrinogen increased clot strength, while the coagulation time (CT) failed to improve after addition of fibrinogen [9,13]. Interestingly, fibrinogen supplementation decreased COT2 
significantly more in haemodilution with HES than in that with RA, while fibrinogen-dependent clot strength increased more in haemodilution with RA. In fact, Fib2 G'max did not increase at all after adding fibrinogen or fibrinogen combined with FXIII in haemodilution with HES. In other words, fibrinogen substitution reversed the early phases of the coagulation regardless of the presence of starch, while haemodilution with HES made fibrinogen unable to improve clot strength. One may speculate that higher doses of fibrinogen may be required to improve also clot strength after HES haemodilution.

FXIII's activity is diminished by haemodilution [39]. FXIII is ineffective at correcting dilutional coagulopathy in vitro [10], but has been shown to decrease postoperative chest-drain bleeding after cardiac surgery [40]. Combining fibrinogen with FXIII in RA haemodilution had an additional on Fib2 G'max but no effect on Fib1 G'max, as discussed above. The additional increase of Fib1 G'max after adding FXIII together with fibrinogen is small (not significant) and will be masked by the platelets when assessing Fib1 G'max. Therefore, analysis of Fib2 G'max will add important information on factors affecting the fibrinogen polymerization.

The findings of this study emphasise the importance of reversing hypothermia and avoiding haemodilution. As previously concluded, it is reasonable to avoid HES in cases of major haemorrhages. Our study showed that the addition of fibrinogen was also beneficial in mild hypothermia and thus, patients are not required to be warmed before infusing fibrinogen. Moreover, if HES has been used in seriously bleeding patients, higher doses than those given in this study are probably needed to reestablish adequate clot strength. Finally, combining fibrinogen with FXIII may be worthwhile in RA induced coagu lopathy, since this combination improved fibrinogendependent clot strength (Fib2 G'max) more than fibrinogen alone in this in vitro study. However, controlled clinical studies need to be undertaken with FOR, to establish correct doses of fibrinogen and factor XIII in dilutional coagulopathy.

In contrast to other studies, we studied the combination of concurrent hypothermia, haemodilution and coagulation factors. We chose to study mild hypothermia and moderate haemodilution, since we wished to study a common and clinically relevant situation. Unfortunately, this decreased the power to detect any statistical differences. Furthermore, FOR is not a widely used method in investigations of coagulopathy in bleeding patients. However, comparison with studies using thrombelastography (ROTEM and TEG) is logical, since FOR is very similar to thrombelastography and these methods are good predictors of perioperative bleeding. There are several other limitations to performing in vitro haemodilution studies using citrated blood, as is the case in this study. The situation in vitro may not accurately represent the situation in vivo. For example, in vitro models do not account for shear stress, release of tissue factor by the endothelium, and activation of procoagulation or fibrinolytic pathways in response to tissue trauma. Our study was performed in blood from healthy volunteers, whereas trauma patients may exhibit a wide range of coagulopathies.

\section{Conclusions}

In conclusion, this in vitro study shows that hypothermia at $33^{\circ} \mathrm{C}$ combined with $33 \%$ haemodilution, especially with HES, interact to impair coagulation variables measured with free oscillation rheometry (FOR). These effects were reduced by adding fibrinogen regardless of hypothermia at $33^{\circ} \mathrm{C}$. After haemodilution with RA, coagulation variables were improved by fibrinogen, but clot strength was not improved with fibrinogen after haemodilution with HES. Further, FXIII had an additional effect on the effects of fibrinogen substitution, at least on the fibrinogendependant clot strength. In summary, higher fibrinogen doses than previously recommended may be required to reverse HES-induced coagulopathy and the combination of fibrinogen with factor XIII may be beneficial. Further clinical investigations are needed to establish the optimal dose of fibrinogen and to elucidate the role of FXIII in dilutional coagulopathy.

\section{Abbreviations}

COT: Clotting time; FXIII: Coagulation factor XIII; Fib1/Fib2: Fibscreen1/ Fibscreen 2 (tests with FOR); FOR: Free oscillation rheometry; G'max: Maximum elasticity; HES: Hydroxyethyl starch; RA: Ringer's acetate solution; ReoRox G2 ${ }^{\circledR}$ : FOR apparatus used in this study; ROTEM ${ }^{\circledR}$ : Rotational thrombelastometry; TEG $^{\circledR}$ : Thrombelastography (both trademark and generic name of technology); VHA: Viscoelastic haemostatic assay e.g., ROTEM, TEG and FOR.

\section{Competing interests}

US has received grants from CSL Beehring. At the time of the study, NT was part time employed by Medirox AB, Nyköping, Sweden. DW and KO have no competing interests.

\section{Authors' contributions}

DW carried out the laboratory work, performed the statistical analysis and drafted the manuscript including all tables and figures. NT drafted the manuscript and participated in the design of tables and figures. $\mathrm{KO}$ drafted the manuscript and participated in the design of tables. US designed the study and drafted the manuscript, and is the senior author of this study. All authors read and approved the final manuscript.

\section{Acknowledgements}

Medirox AB (Nyköping, Sweden) kindly provided the Reorox G2 (FOR) instrument and test materials. The study was partly funded by Region Skåne (Sweden).

\section{Author details}

'Consultant Anaesthetist, Lund University, Skane Universisty Hospital, Lund, 22185 Lund, Sweden. ${ }^{2}$ Division of Transfusion Medicine, Department of Clinical and Experimental Medicine, Faculty of Health Sciences, Linköping University, Linköping, Sweden. ${ }^{3}$ Department of Clinical Immunology and Transfusion Medicine, County Council of Östergötland, Linköping, Sweden.

Received: 6 November 2012 Accepted: 12 March 2013

Published: 22 March 2013 
References

1. Sauaia A, Moore FA, Moore EE, Moser KS, Brennan R, Read RA, Pons PT: Epidemiology of trauma deaths: a reassessment. J Trauma 1995, 38:185-193.

2. Soreide $K$, Kruger AJ, Vardal AL, Ellingsen $C L$, Soreide E, Lossius HM: Epidemiology and contemporary patterns of trauma deaths: changing place, similar pace, older face. World J Surg 2007, 31:2092-2103.

3. Bolliger DMD, Gorlinger KMD: Tanaka KAMDMS: Pathophysiology and Treatment of Coagulopathy in Massive Hemorrhage and Hemodilution. Anesthesiology 2010, 113:1205-1219.

4. Johansson $\mathrm{Pl}$, Ostrowski $\mathrm{SR}$, Secher $\mathrm{NH}$ : Management of major blood loss: an update. Acta Anaesthesiol Scand 2010, 54:1039-1049.

5. Coats TJ, Brazil E, Heron M: The effects of commonly used resuscitation fluids on whole blood coagulation. Emerg Med J 2006, 23:546-549.

6. Egli GA, Zollinger A, Seifert B, Popovic D, Pasch T, Spahn DR: Effect of progressive haemodilution with hydroxyethyl starch, gelatin and albumin on blood coagulation. Br J Anaesth 1997, 78:684-689.

7. Hiippala ST, Myllyla GJ, Vahtera EM: Hemostatic factors and replacement of major blood loss with plasma-poor red cell concentrates. Anesth Analg 1995, 81:360-365.

8. Rossaint R, Bouillon B, Cerny V, Coats TJ, Duranteau J, Fernandez-Mondejar E, Hunt BJ, Komadina R, Nardi G, Neugebauer E, et al: Management of bleeding following major trauma: an updated European guideline. Crit Care 2010, 14:R52.

9. Fenger-Eriksen C, Anker-Moller E, Heslop J, Ingerslev J, Sorensen B: Thrombelastographic whole blood clot formation after ex vivo addition of plasma substitutes: improvements of the induced coagulopathy with fibrinogen concentrate. Br J Anaesth 2005, 94:324-329.

10. Haas T, Fries D, Velik-Salchner $C$, Reif $C$, Klingler A, Innerhofer $P$ : The in vitro effects of fibrinogen concentrate, factor XIII and fresh frozen plasma on impaired clot formation after 60\% dilution. Anesth Analg 2008, 106:1360-1365.

11. Fenger-Eriksen $C$, Jensen TM, Kristensen BS, Jensen KM, Tonnesen $E$, Ingerslev J, Sorensen B: Fibrinogen substitution improves whole blood clot firmness after dilution with hydroxyethyl starch in bleeding patients undergoing radical cystectomy: a randomized, placebo-controlled clinical trial. J Thromb Haemost 2009, 7:795-802.

12. Fries D, Haas T, Klingler A, Streif W, Klima G, Martini J, Wagner-Berger H, Innerhofer P: Efficacy of fibrinogen and prothrombin complex concentrate used to reverse dilutional coagulopathy-a porcine model. Br J Anaesth 2006, 97:460-467.

13. Fries D, Krismer A, Klingler A, Streif W, Klima G, Wenzel V, Haas T, Innerhofer $P$ : Effect of fibrinogen on reversal of dilutional coagulopathy: a porcine model. Br J Anaesth 2005, 95:172-177.

14. Borgman MA, Spinella PC, Perkins JG, Grathwohl KW, Repine T, Beekley AC, Sebesta J, Jenkins D, Wade CE, Holcomb JB: The ratio of blood products transfused affects mortality in patients receiving massive transfusions at a combat support hospital. J Trauma 2007, 63:805-813.

15. Shaz BH, Dente CJ, Nicholas J, MacLeod JB, Young AN, Easley K, Ling Q Harris RS, Hillyer CD: Increased number of coagulation products in relationship to red blood cell products transfused improves mortality in trauma patients. Transfusion 2010, 50:493-500

16. Nielsen VG: Colloids decrease clot propagation and strength: role of factor XIII-fibrin polymer and thrombin-fibrinogen interactions. Acta Anaesthesio/ Scand 2005, 49:1163-1171.

17. Ganter MT, Hofer CK: Coagulation monitoring: current techniques and clinical use of viscoelastic point-of-care coagulation devices. Anesth Analg 2008, 106:1366-1375.

18. Rundgren M, Engstrom M, MD P: A thromboelastometric evaluation of the effects of hypothermia on the coagulation system. Anesth Analg 2008, 107:1465-1468.

19. Spiel AO, Kliegel A, Janata A, Uray T, Mayr FB, Laggner AN, Jilma B, Sterz F: Hemostasis in cardiac arrest patients treated with mild hypothermia initiated by cold fluids. Resuscitation 2009, 80:762-765.

20. Lier H, Böttiger BW, Hinkelbein J, Krep H, Bernhard M: Coagulation management in multiple trauma: a systematic review. Intensive Care Med 2011, 37:572-582.

21. Tynngard N, Lindahl T, Ramstrom S, Berlin G: Effects of different blood components on clot retraction analysed by measuring elasticity with a free oscillating rheometer. Platelets 2006, 17:545-554.

22. Rånby $M$, Ramström $S$, Svensson PO, Lindahl TL: Clotting time by free oscillation rheometry and visual inspection and a viscoelastic description of the clotting phenomenon. Scand J Clin Lab Invest 2003, 63:397.
23. Tynngard N, Lindahl TL, Ramstrom S, Raf T, Rugarn O, Berlin G: Free oscillation rheometry detects changes in clot properties in pregnancy and thrombocytopenia. Platelets 2008, 19:373-378.

24. Wobbrock JO, Findlater L, Gergle D, Higgins JJ: The Aligned Rank Transform for nonparametric factorial analyses using only ANOVA procedures. In Proceedings of the ACM Conference on Human Factors in Computing Systems (CHI '11); May 7-12, 2011; Vancouver, British Columbia. Canada: ACM Press; 2011:143-146.

25. Martini WZ, Cortez DS, Dubick MA, Park MS, Holcomb JB: Thrombelastography is better than PT, aPTT, and activated clotting time in detecting clinically relevant clotting abnormalities after hypothermia, hemorrhagic shock and resuscitation in pigs. J Trauma 2008, 65:535-543.

26. Dirkmann D, Hanke AA, Görlinger K, Peters J: Hypothermia and acidosis synergistically impair coagulation in human whole blood. Anesth Analg 2008, 106:1627-1632

27. Martini WZ: The effects of hypothermia on fibrinogen metabolism and coagulation function in swine. Metabolism 2007, 56:214-221.

28. Konrad CJ, Markl TJ, Schuepfer GK, Schmeck J, Gerber HR: In vitro effects of different medium molecular hydroxyethyl starch solutions and lactated ringer's solution on coagulation using SONOCLOT. Anesth Analg 2000, 90:274.

29. Godier A, Durand M, Smadja D, Jeandel T, Emmerich J, Samama CM: Maizeor potato-derived hydroxyethyl starches: is there any thromboelastometric difference? Acta Anaesthesio/ Scand 2010, 54:1241-1247.

30. Mittermayr M, Streif W, Haas T, Fries D, Velik-Salchner C, Klingler A, Oswald E, Bach C, Schnapka-Koepf M, Innerhofer P: Hemostatic changes after crystalloid or colloid fluid administration during major orthopedic surgery: the role of fibrinogen administration. Anesth Analg 2007, 105:905-917.

31. Schramko A, Suojaranta-Ylinen R, Kuitunen A, Raivio P, Kukkonen S, Niemi T: Hydroxyethylstarch and gelatin solutions impair blood coagulation after cardiac surgery: a prospective randomized trial. Br J Anaesth 2010, 104:691-697.

32. Wohner N: Role of cellular elements in thrombus formation and dissolution. Cardiovasc Hematol Agents Med Chem 2008, 6:224-228.

33. Bochsen L: The influence of platelets, plasma and red blood cells on functional haemostatic assays. Blood Coagul Fibrinolysis 2011, 22:167-175.

34. Ogawa S, Szlam F, Bolliger D, Nishimura T, Chen EP, Tanaka KA: The impact of hematocrit on fibrin clot formation assessed by rotational thromboelastometry. Anesth Analg 2012, 115:16-21.

35. Darlington DN, Kremenevskiy I, Pusateri AE, Scherer MR, Fedyk CG, Kheirabaldi BS, Delgado AV, Dubick MA: Effects of In vitro hemodilution, hypothermia and rFVIla addition on coagulation in human blood. Int J Burns Trauma 2012, 2:42-50.

36. Gubler KD, Gentilello LM, Hassantash SA, Maier RV: The impact of hypothermia on dilutional coagulopathy. J Trauma 1994, 36:847-851.

37. Karlsson M, Ternstrom L, Hyllner M, Baghaei F, Flinck A, Skrtic S, Jeppsson A: Prophylactic fibrinogen infusion reduces bleeding after coronary artery bypass surgery. A prospective randomised pilot study. Thromb Haemost 2009, 102:137-144

38. Stinger HK, Spinella PC, Perkins JG, Grathwohl KW, Salinas J, Martini WZ, Hess JR, Dubick MA, Simon CD, Beekley AC, et al: The ratio of fibrinogen to red cells transfused affects survival in casualties receiving massive transfusions at an army combat support hospital. J Trauma 2008,64:S79-S85.

39. Ternström L, Radulovic V, Karlsson M, Baghaei F, Hyllner M, Bylock A, Hansson KM, Jeppsson A: Plasma activity of individual coagulation factors, hemodilution and blood loss after cardiac surgery: a prospective observational study. Thromb Res 2010, 126:e128-e133.

40. Levy JH, Gill R, Nussmeier NA, Olsen PS, Andersen HF, Booth FV, Jespersen CM: Repletion of factor XIII following cardiopulmonary bypass using a recombinant A-subunit homodimer. A preliminary report. Thromb Haemost 2009, 102:765-771.

doi:10.1186/1757-7241-21-20

Cite this article as: Winstedt et al:: Free oscillation rheometry monitoring of haemodilution and hypothermia and correction with fibrinogen and factor XIII concentrates. Scandinavian Journal of Trauma, Resuscitation and Emergency Medicine 2013 21:20 